\title{
COMMENTARIES
}

\section{Culture clash: challenging the dogma of microbial diversity}

\author{
Stuart P Donachie, Jamie S Foster and Mark V Brown
}

The ISME Journal (2007) 1, 97-99; doi:10.1038/ismej.2007.22; published online 10 May 2007

The advent of ribosomal RNA sequence analysis 30 years ago revolutionized microbial ecology and significantly expanded our knowledge of microbial phylogenetic diversity (Woese and Fox, 1977). However, exclusive reliance on this single approach in environmental studies perpetuates certain assumptions that should be discussed and re-evaluated. These assumptions include (1) ribosomal RNA gene analyses can be used exclusively for the study of microbial diversity; (2) the number of colonyforming units (CFU) on nutrient media as a fraction of the total number of bacteria determined by microscopy is related to phylogenetic diversity and (3) only a fraction of environmental bacteria 'species' are culturable. Overlooking a century of cultivation history and encouraging use only of ribosomal approaches leads to significant gaps in microbial community diversity data. We demonstrate that cultivation methods are critical in microbial diversity studies and that they detect organisms undetected by molecular techniques. We thus caution against single-method approaches and posit that metagenomic techniques may be complemented by parallel culture libraries whenever diversity information is sought.

Use of the ribosomal approach alone in diversity studies originates in part with a review of its role in microbial ecology by Olsen et al. (1986) who stated, '...because molecules rather than organisms are isolated, the method is not limited to species that are amenable to laboratory cultivation'. Indeed, this technique has detected numerous microorganisms, for which no cultivated representatives are known (Stahl et al., 1984; Pace et al., 1986; Ward et al., 1990). Since these landmark studies, it has become a common practice to extract community DNA, amplify and clone fragments of ribosomal RNA genes and generate nucleotide sequences for comparison with those retrieved by other such studies. However, cultivation approaches are often set aside as researchers purport to describe microbial diversity by cultivating nothing more than a plasmid.

Central to this unilateral approach is the general opinion that since cultivation approaches detect only a fraction of the total number of bacteria in a sample such techniques have little value or are 'limited in scope' (Fry, 2000). This opinion appears rooted in the 'great plate count anomaly' (Staley and Konopka, 1985), whereby CFU counts are described as a small fraction of the total number of cells determined by nucleic acid staining and direct microscopy. A percentage range for such fractions reported by Amann et al. (1995) is often cited to support the contention that cultivation approaches detect little diversity while molecular-based approaches provide a more comprehensive view. However, neither CFU counts nor cell abundance determined by direct microscopy offer any phylogenetic information at all, that is, CFU fractions amounting to $0.001-15 \%$ of the total count are not related to the number of 'species' present. Simply put, since these techniques measure cell abundance, the CFU fraction of the direct microscopic count thus has no relevance or value in selecting the ribosomal approach alone to define diversity in terms of the number of 'species' present.

A recent review of metagenomics also notes that the discrepancy between plate and direct counts, '... is dramatic in some aquatic environments... and in soil [from which] 0.1 to $1 \%$ of bacteria are readily culturable on common media under standard conditions' (Handelsman, 2004). Such statements perpetuate the idea that only $1 \%$ of soil and marine microbial species are 'culturable' and that one can opt instead to describe diversity exclusively via a molecular approach. Given that 100 years have passed since the Delft School pioneered the use of diverse media and incubation conditions to isolate specific microbes, those versed in cultivation methods must ponder, 'What are common media under standard conditions?' How much can we reasonably expect one medium to tell us of the phylogenetic diversity or the culturability of bacteria in a sample? Just as molecular biologists do not use one universal method to isolate community DNA, or one PCR routine or sequencing technology, practitioners of cultivation techniques investigating diversity use diverse inocula dilutions, culture media and $\mathrm{pH}$, incubation temperatures and atmospheres. Such conditions increase the metabolic diversity of, and by definition, the number of species cultivated from a sample.

To address these points, we compared studies, in which both 16S rDNA sequencing and cultivation 


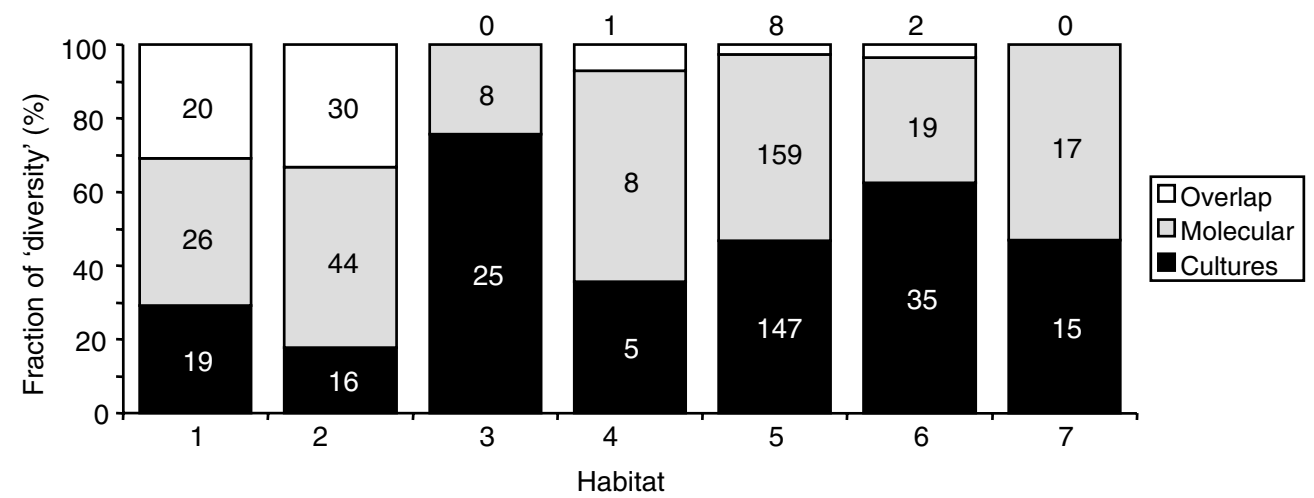

Figure 1 Comparative analysis of diversity detected by contemporaneously applied culture-dependent and culture-independent approaches in the same habitat. The numbers of unique sequence groups represent those reported in libraries compiled through molecular analysis (PCRbased 16S rRNA clones) or cultivations. If a 'species' was detected by both methods it was listed separately ('overlap'). OTU and culture group numbers are presented here as reported in the original paper. The seven habitats shown are (1) endodontic pathogenic oral biofilms (Munson et al., 2002); (2) exodontic oral biofilms (Munson et al., 2004); (3) Hawaiian lakes and Lō'ihi (Donachie et al., 2004a); (4) rhizoplane (Kaiser et al., 2001); (5) bird feathers (Shawkey et al., 2005); (6) ocean (Eilers et al., 2000); (7) hypersaline salterns (Maturrano et al., 2006).

techniques were applied to the same sample (Figure 1). A crucial point we address is the extent to which the datasets of cultivation-dependent and independent approaches overlap, that is, how many of the same taxa are detected by both techniques? Since the ribosomal rRNA gene approach is not limited to those species in culture (Olsen et al., 1986) it should detect all microbes in the habitat, both 'culturable' and 'unculturable'. If this is indeed the case, culture libraries should not detect taxa absent from datasets recovered via the ribosomal approach. Such assumptions, however, often do not hold true. One such study of microbial diversity in Hawaiian lakes and at the Hawaiian submarine volcano Lō'ihi, recorded 158 operational taxonomic units (OTUs) from 18 Bacteria phyla, with seven unassigned and two Euryarchaeota (Donachie et al., 2004a), where sequences in each OTU shared $\geqslant 97 \%$ 16S rDNA insert sequence identity. Bacteria cultivated from the same samples were grouped into 155 'taxa' from 10 phyla, with cultures in each taxon sharing $\geqslant 97 \% 16 \mathrm{~S}$ rDNA sequence identity. Although the number of taxa recorded by each method is comparable, the datasets had little in common, with eight OTUs and cultures from the same samples sharing $\geqslant 97 \%$ sequence identity (Figure 1). Of particular importance to diversity studies, 55 cultivated taxa shared $<97 \%$ identity with published sequences, and 52 of these from seven phyla were absent from the study's clone libraries. The defining phenotypic traits of four new species were not detected by the ribosomal approach (Donachie et al., 2003, 2004b, 2005, 2006). Cultivating so many Bacteria not represented in a clone library demonstrates that a significant fraction of diversity would have been overlooked had only $16 \mathrm{~S}$ rDNA clone libraries been analyzed. We thus cannot conclude that the molecular approach captured the 'full spectrum of microbial diversity'.

One challenge is to determine why so many cultivated microorganisms are not detected in $16 \mathrm{~S}$ rDNA clone libraries. We posit that although there may not be one consistent cause, preparation steps in the molecular approach may introduce some biases. For example, DNA might not be extracted from difficult to lyse cells (for example, Firmicutes) during community DNA extraction (Donachie et al., 2004a). Conversely, these authors dismissed a 'lack of primer specificity' because pure cultures in their study were sequenced with the same primers used in clone library construction. Others have reported that early and key steps in the ribosomal approach such as those pertaining to PCR conditions and template to product ratios affect the composition of the resultant clone libraries (Mathieu-Daudé et al., 1996; Suzuki and Giovannoni, 1996; Polz and Cavanaugh, 1998; Bonnet et al., 2002; Hongoh et al., 2003). In addition, high DNA sequencing costs until recently meant analysis of small sequences and small, unrepresentative libraries was tacitly accepted (Hill et al., 2002). It remains to be seen if high-throughput cultivation and sequencing techniques will enable 'molecular methods' to fulfill the promise of detecting all microbes in a community, or if we really never will know the true extent of microbial diversity (Curtis et al., 2002).

Papers in which cultivation techniques and ribosomal sequence analysis are applied contemporaneously appear regularly in the diversity literature, but differences between the libraries are often disregarded. Exclusive usage of the ribosomal approach overlooks a significant fraction of phylogenetic diversity easily determined by cultivation methods. These missing culturable fractions are well above $1 \%$ when the number of OTUs and distinct cultures is considered as $100 \%$ (Figure 1). These data support use of a polyphasic approach to describe microbial diversity. As it has been suggested that we refrain from using the term 'prokaryote' because it is 'outdated' and might mislead our students (Pace, 2006), a similar plea should be entered for statements that suggest cultivation methods describe just $1 \%$ of diversity, and that 
molecular methods alone can detect 'unculturable' (i.e., previously uncultivated) bacteria. The data summarized here demonstrate that culture-dependent techniques deserve more credit. Only by implementing comprehensive strategies that include parallel culturing and molecular techniques can we begin to understand the full extent of microbial diversity within a community (Hengstmann et al., 1999; Oremland et al., 2005).

SP Donachie is at Department of Microbiology, University of Hawai'i, Honolulu, HI, USA; JS Foster is at Department of Microbiology and Cell Science, University of Florida Space Life Science Laboratory, Kennedy Space Center, Kennedy, FL, USA and MV Brown is at NASA Astrobiology Institute at the University of Hawai'i, Institute for Astronomy, Honolulu, HI, USA. E-mail: donachie@hawaii.edu

\section{References}

Amann RI, Ludwig W, Schleifer KH. (1995). Phylogenetic identification and in situ detection of individual microbial cells without cultivation. Microbiol Rev 59: 143-169.

Bonnet R, Suau A, Dore J, Gibson GR, Collins MD. (2002). Differences in rDNA libraries of faecal bacteria derived from 10- and 25-cycle PCRs. Int J Syst Evol Microbiol 52: $757-763$.

Curtis TP, Sloan WT, Scannell JW. (2002). Estimating prokaryotic diversity and its limits. Proc Natl Acad Sci USA 99: 10494-10499.

Donachie SP, Bowman JP, Alam M. (2004b). Psychroflexus tropicus, sp. nov., a new, obligately halophilic CFB group bacterium isolated from an Hawaiian hypersaline lake. Int J Syst Evol Microbiol 54: 935-940.

Donachie SP, Bowman JP, Alam M. (2006). Nesiotobacter exalbescens gen. nov., sp. nov., a moderately thermophilic alphaproteobacterium from an Hawaiian hypersaline lake. Int J Syst Evol Microbiol 56: 563-567.

Donachie SP, Bowman JP, On SL, Alam M. (2005). Arcobacter halophilus sp. nov., the first obligate halophile in the genus Arcobacter. Int J Syst Evol Microbiol 55: 1271-1277.

Donachie SP, Hou S, Gregory TS, Malahoff A, Alam M. (2003). Idiomarina loihiensis, sp. nov., a new halophilic $\gamma$-Proteobacterium isolated from the Lō'ihi submarine volcano, Hawai'i. Int J Syst Evol Microbiol 53: 1873-1879.

Donachie SP, Hou S, Lee K-S, Riley CW, Pikina A, Belisle C et al. (2004a). The Hawaiian Archipelago: a microbial diversity hotspot. Microb Ecol 48: 509-520.

Eilers H, Pernthaler J, Glöckner FO, Amann R. (2000). Culturability and in situ abundance of pelagic bacteria from the North Sea. Appl Environ Microbiol 66: 3044-3051.

Fry J. (2000). Bacterial diversity and 'unculturables'. Microbiol Today 27: 186-188.

Handelsman J. (2004). Metagenomics: Application of genomics to uncultured microorganisms. Microbiol Mol Biol Rev 68: 669-685.
Hengstmann U, Chin KJ, Janssen PH, Liesack W. (1999). Comparative phylogenetic assignment of environmental sequences of genes encoding $16 \mathrm{~S}$ rRNA and numerically abundant culturable bacteria from an anoxic rice paddy soil. Appl Environ Microbiol 65: 5050-5058.

Hill JE, Seipp RP, Betts M, Hawkins L, Van Kessel AG, Crosby WL et al. (2002). Extensive profiling of a complex microbial community by high-throughput sequencing. Appl Environ Microbiol 68: 3055-3066.

Hongoh Y, Yuzawa H, Ohkuma M, Kudo T. (2003). Evaluation of primers and PCR conditions for the analysis of $16 \mathrm{~S}$ rRNA genes from a natural environment. FEMS Microbiol Lett 221: 299-304.

Kaiser O, Pühler A, Selbitschka W. (2001). Phylogenetic analysis of microbial diversity in the rhizoplane of Oilseed Rape (Brassica napus cv. Westar) employing cultivation-dependent and cultivation-independent approaches. Microb Ecol 42: 136-149.

Mathieu-Daudé F, Welsh J, Vogt T, McClelland M. (1996). DNA rehybridization during PCR: the ' $\mathrm{C}_{\mathrm{o}} \mathrm{t}$ effect' and its consequences. Nucl Acids Res 24: 2080-2086.

Maturrano L, Santos F, Rosselló-Mora R, Antón J. (2006). Microbial diversity in Maras salterns, a hypersaline environment in the Peruvian Andes. Appl Environ Microbiol 72: 3887-3895.

Munson MA, Banerjee A, Watson TF, Wade WG. (2004). Molecular analysis of the microflora associated with dental caries. J Clin Microbiol 42: 3023-3029.

Munson MA, Pitt-Ford T, Chong B, Weightman A, Wade WG. (2002). Molecular and cultural analysis of the microflora associated with endodontic infections. J Dent Res 81: 761-766.

Olsen GJ, Lane DJ, Giovannoni SJ, Pace NR, Stahl DA. (1986). Microbial ecology and evolution: a ribosomal RNA approach. Annu Rev Microbiol 40: 337-365.

Oremland RS, Capone DG, Stolz JF, Fuhrman J. (2005). Whither or wither geomicrobiology in the era of 'community metagenomics'. Nat Rev Microbiol 3: 572-578.

Pace NR, Stahl DA, Lane DJ, Olsen GJ. (1986). The analysis of natural microbial populations by ribosomal RNA sequences. Adv Microb Ecol 9: 1-55.

Pace N. (2006). A time for change. Nature 441: 289.

Polz MF, Cavanaugh CM. (1998). Bias in templateto-product ratios in multi-template PCR. Appl Environ Microbiol 64: 3724-3730.

Shawkey MD, Mills KL, Dale C, Hill GE. (2005). Microbial diversity of wild bird feathers revealed through cultured-based and culture-independent techniques. Microb Ecol 50: 40-47.

Stahl DA, Lane DJ, Olsen GJ, Pace NR. (1984). Analysis of hydrothermal vent-associated symbionts by ribosomal RNA sequences. Science 224: 409-411.

Staley JT, Konopka A. (1985). Measurement of in situ activities of non-photosynthetic microorganisms in aquatic and terrestrial habitats. Annu Rev Microbiol 39: 321-346.

Suzuki MT, Giovannoni SJ. (1996). Bias caused by template annealing in the amplification of mixtures of 16S rRNA genes by PCR. Appl Environ Microbiol 62: 625-630.

Ward DM, Weller R, Bateson MM. (1990). 16S rRNA sequences reveal numerous uncultured microorganisms in a natural community. Nature 345: 63-65.

Woese C, Fox G. (1977). Phylogenetic structure of the prokaryotic domain: the primary kingdoms. Proc Natl Acad Sci USA 74: 5088-5090. 